\title{
Trends in hospital admissions for pulmonary embolism in Spain from 2002 to 2011
}

\author{
Javier de Miguel-Díez', Rodrigo Jiménez-García², David Jiménez³, \\ Manuel Monreal ${ }^{4}$, Ricardo Guijarro ${ }^{5}$, Remedios Otero6, \\ Valentín Hernández-Barrera², Javier Trujillo-Santos ${ }^{7}$, \\ Ana López de Andrés² and Pilar Carrasco-Garrido²
}

Affiliations: 'Pneumology Dept, Hospital General Universitario Gregorio Marañon, Madrid, Spain. ${ }^{2}$ Preventive Medicine and Public Health Teaching and Research Unit, Dept of Health Sciences, Universidad Rey Juan Carlos, Madrid, Spain. ${ }^{3}$ Respiratory Dept, Ramón y Cajal Hospital, IRYCIS, Madrid, Spain. ${ }^{4}$ Dept of Internal Medicine, Hospital Universitari Germans Trias i Pujol, Badalona, Spain. ${ }^{5}$ Dept of Internal Medicine, Hospital Carlos Haya, Malaga, Spain. ${ }^{6}$ Medical-Surgical Unit of Respiratory Diseases, University Hospital Virgen del Rocio, Seville, Spain. ${ }^{7}$ Dept of Medicine, Hospital Universitario Santa Lucía, Cartagena, Spain.

Correspondence: Javier de Miguel-Díez, Pneumology Dept, Hospital General Universitario Gregorio Marañón, C/Doctor Esquerdo 46, 28007 Madrid, Spain. E-mail: javier.migueldasalud.madrid.org

ABSTRACT The aim of our study was to analyse changes in the incidence, diagnostic procedures, comorbidity, length of hospital stay, costs and in-hospital mortality of patients hospitalised for pulmonary embolism in Spain over a 10-year period.

We included all patients who were hospitalised for pulmonary embolism (ICD-9-CM codes 415.11 and 415.19) as the primary diagnosis between 2002 and 2011. Data were collected from the National Hospital Discharge Database, covering the entire Spanish population.

115671 patients were admitted. The overall crude incidence increased from 20.44 per 100000 inhabitants in 2002 to 32.69 in $2011(\mathrm{p}<0.05)$. In 2002, 13.3\% of patients had a Charlson comorbidity index $>2$, and in 2011 the prevalence increased to $20.8 \%$ ( $\mathrm{p}<0.05$ ). Mean length of hospital stay was 12.7 days in 2002 and decreased to 9.99 in $2011(\mathrm{p}<0.05)$. During the study period, mean cost per patient increased from $€ 3915$ to $€ 4372(\mathrm{p}<0.05)$. In-hospital mortality decreased from $12.9 \%$ in 2002 to $8.32 \%$ in $2011(\mathrm{p}<0.05)$. The increase in the use of computed tomographic pulmonary angiography over time was associated with increased incidence and lower mortality.

Our results revealed an increase in the incidence of hospitalised pulmonary embolism patients from 2002 to 2011 with concomitant increase in comorbidities and cost. However, length of hospital stay and inhospital mortality decreased.

@ERSpublications

Increase in hospital admission rates and decrease in pulmonary embolism mortality over time in Spain http://ow.ly/xEmVu 


\section{Introduction}

Pulmonary embolism is a major public health problem. Although less common than other vascular diseases, such as myocardial infarction or stroke, it is also a severe disease [1]. Patients with acute pulmonary embolism usually require hospitalisation $[1,2]$. In addition to significant morbidity and mortality, the economic burden associated with pulmonary embolism is very high [3].

Collecting data on hospitalisations for pulmonary embolism is important at a national level to evaluate incidence, patient characteristics and outcomes. For instance, a change has recently been observed in the epidemiological patterns of pulmonary embolism in the USA. WIENER et al. [4] found an increased incidence, minimal changes in mortality and a substantially lower case fatality rate. Hospital admission rates for pulmonary embolism have also increased over time in the UK [5] and Australia [6]. During recent years, pulmonary embolism mortality has also decreased in these countries [5,6]. Meanwhile, in other countries, such as China, the incidence of pulmonary embolism has remained relatively stable in recent years while the case fatality rate has declined over the same period [7]. International variation may have been partly due to different risk factors for pulmonary embolism or inconsistencies in the accuracy of diagnosis.

Comparing hospital admissions and outcomes for pulmonary embolism between countries could help each country understand their data set and could also aid in planning healthcare services provision. The National Hospital Discharge Database provides a large alternative information source to describe and analyse the trends and characteristics of hospitalisations for pulmonary embolism at a national level.

Little is known about the epidemiology of pulmonary embolism in Spain [8, 9]. In recent years, the RIETE (Registro Informatizado de la Enfermedad TromboEmbólica) registry has shed light on the care and management of pulmonary embolism in Spain [10], but the registry was not designed for the evaluation of pulmonary embolism epidemiology. Thus, there are no recent epidemiological studies in Spain, and national estimates are extrapolated from other countries. However, it is worth remembering that results of epidemiological studies cannot be extrapolated to other populations.

The aim of this study was to conduct a nationwide analysis of discharge data, collected from 2002 to 2011 years. These data were used to elucidate changes in the incidence, diagnostic procedures, comorbidity profiles, length of hospital stay, economic costs and in-hospital mortality of patients hospitalised for pulmonary embolism in Spain over a 10-year study period.

\section{Methods}

According to the Spanish Health System, at the time of discharge of each hospitalisation physicians must declare all diagnoses and procedures performed, using the International Classification of Disease, 9th revision codes (ICD-9-CM). This information is collected by the Spanish National Hospital Database, namely Conjunto Minimo Basico de Datos (CMBD), which compiles all the hospital data of the Spanish National Health System [11]. The CMBD database includes patients' variables (sex and date of birth), date of admittance, date of discharge, discharge destination (home, deceased or other health/social institution), and details of up to 14 discharge diagnoses and up to 20 procedures performed during admission.

We selected all patients hospitalised for pulmonary embolism (ICD-9-CM codes 415.11 and 415.19) as the primary diagnosis during 2002-2011. We calculated the annual age-specific incidence proportions by dividing the number of cases per year per age group, by the corresponding number of people in that population group according to the National Institute of Statistics reported at December 31st each year [12]. The incidence proportions were expressed in terms of 100000 inhabitants. The proportion of patients that died during hospital admission (in-hospital mortality), length of hospital stay and costs were also estimated for each year studied. Costs were calculated using diagnosis-related groups for the disease. Diagnosis-related groups represent a medical economic entity concerning a set of diseases requiring analogous management resources [13]. All costs shown were adjusted for the increment of the inflation in the same period in Spain. Clinical characteristics included information on overall comorbidity at the time of the hospitalisation, which was assessed by computing the Charlson comorbidity index [14]. The index applies to 17 disease categories that are totalled to obtain an overall score for each patient. We divided patients into three categories: 1) low index, which corresponds to patients with no previously recorded disease categories in the Charlson comorbidity index; 2) medium index, patients with one or two disease categories; and 3) high index, patients with more than two disease categories. For the purpose of the study we also identified patients who suffered from cancer as a concomitant disease using the codes included in the Charlson comorbidity index [14].

We specifically identified the following procedures: computed tomographic pulmonary angiography (CTPA), lung scintigraphy and any surgical process. 


\section{Statistical analysis}

Quantitative variables were expressed as mean \pm SD. Qualitative variables were expressed as frequencies and percentages. Comparisons were performed using the Chi-squared test, Fisher's exact test, t-test or ANOVA as appropriate. Multivariate analysis of time trend of study variables was conducted using Poisson regression models for incidence and logistic regression models for in-hospital mortality and adjusting for age, sex and other covariables. We then checked for interactions between the independent variables in the regression models. Estimates were made using STATA version 10.1 (StataCorp LP, College Station, TX, USA) and statistical significance was set at two-tailed $\alpha<0.05$.

Data were treated with full confidentiality according to the Spanish legislation. Patient identifiers were removed before the database was provided to the authors in order to keep strict patient confidentiality. It was not possible to identify individual patients in this study or in the database. Given the anonymous and mandatory nature of the dataset, it was not necessary to obtain informed consent. The Spanish Ministry of Health evaluated the protocol of our investigation and considered that it met all ethical aspects according to the Spanish legislation so provided us with the anonymous database. In addition, the study protocol was approved by the ethics committee of the Universidad Rey Juan Carlos (Madrid, Spain).

\section{Results}

In total, we identified a total of 115671 discharges of patients (52 856 males and 62815 females) admitted for pulmonary embolism as the primary diagnosis in Spain from 2002 to 2011 . Table 1 shows age-specific incidence proportions and distribution according to sex. The overall crude incidence increased from 20.4 hospitalisations to 32.7 hospitalisations for pulmonary embolism per 100000 inhabitants in Spain from 2002 to $2011(\mathrm{p}<0.05)$. Incidence proportions were higher in older patients, but there was a significant increase over time in all age groups. However, no significant differences were found in sex distribution during the study period.

Mean length of hospital stay for admissions for pulmonary embolism was 12.7 days in 2002, decreasing to 9.99 days in 2011. Time trends in Charlson comorbidity index are summarised in table 2. In 2002, 45.0\% of patients had a Charlson comorbidity index of $0,41.7 \%$ had a Charlson comorbidity index of $1-2$, and $13.3 \%$ had a Charlson comorbidity index of $>2$. In 2011, the proportion of patients who had been hospitalised for pulmonary embolism and had a Charlson comorbidity index of 1-2 or $>2$ had increased to $42.5 \%$ and $20.8 \%$, respectively. There is a significant association between the Charlson comorbidity index and year $(\mathrm{p}<0.001)$. The proportion of pulmonary embolism patients who had cancer increased significantly throughout the study, from $11.8 \%$ in 2002 to $17.5 \%$ in 2011 . Conversely, the proportion of pulmonary embolism patients who had undergone surgical treatment did not change over time, from $2.7 \%$ in 2002 to $2.14 \%$ in 2011. However, the mean cost per patient increased from $€ 3915$ in 2002 to $€ 4372$ in 2011.

Table 3 shows the diagnostic tests performed in patients hospitalised for pulmonary embolism during the study period for the total sample and according to the presence of cancer. There was a significant increase in the use of CTPA, from $32.1 \%$ in 2002 to $64.4 \%$ in 2011 . This trend was observed in patients with and without cancer. In parallel, the use of lung scintigraphy decreased over the same period, from $42.9 \%$ in 2002 to $7.8 \%$ in 2011 . In patients who died in hospital, imaging tests were also carried out, although in a smaller proportion than in those who survived. In both cases, the use of CTPA increased over time and the use of lung scintigraphy decreased (table 4). Mean in-hospital mortality decreased from $12.9 \%$ in 2002 to $8.32 \%$ in 2011.

Table 5 summarises the results of multivariate analysis of trends and factors associated with incidence and in-hospital death among patients hospitalised for pulmonary embolism. After controlling for possible confounders using Poisson regression models, there was a significant increase in incidence from 2002 to 2011. Factors associated with incidence included age, sex and Charlson comorbidity index. Thereby, the incidence was higher in older subjects, females and patients with $>2$ comorbidities. With regard to inhospital mortality, after adjusting the logistic regression model, there was a significant decrease in mortality from 2002 to 2011. The risk of in-hospital mortality was higher in females, older patients, and patients with increasing Charlson comorbidity index. Regarding diagnostic procedures, multivariate analysis showed an increase in the use of CTPA over time, which was associated with increased incidence and lower mortality. Other procedures such as lung scintigraphy were performed progressively less frequently, which is associated with a lower incidence and lower mortality.

\section{Discussion}

In this observational study including 115671 hospital admissions for pulmonary embolism, we found a significant increase in incidence proportions of hospitalisations from 2002 to 2011. Hospital admissions rates for pulmonary embolism have also increased in the USA [15] and Denmark [16], as well as in other countries, such as those mentioned previously [3-5]. In contrast, we found a decrease in pulmonary 


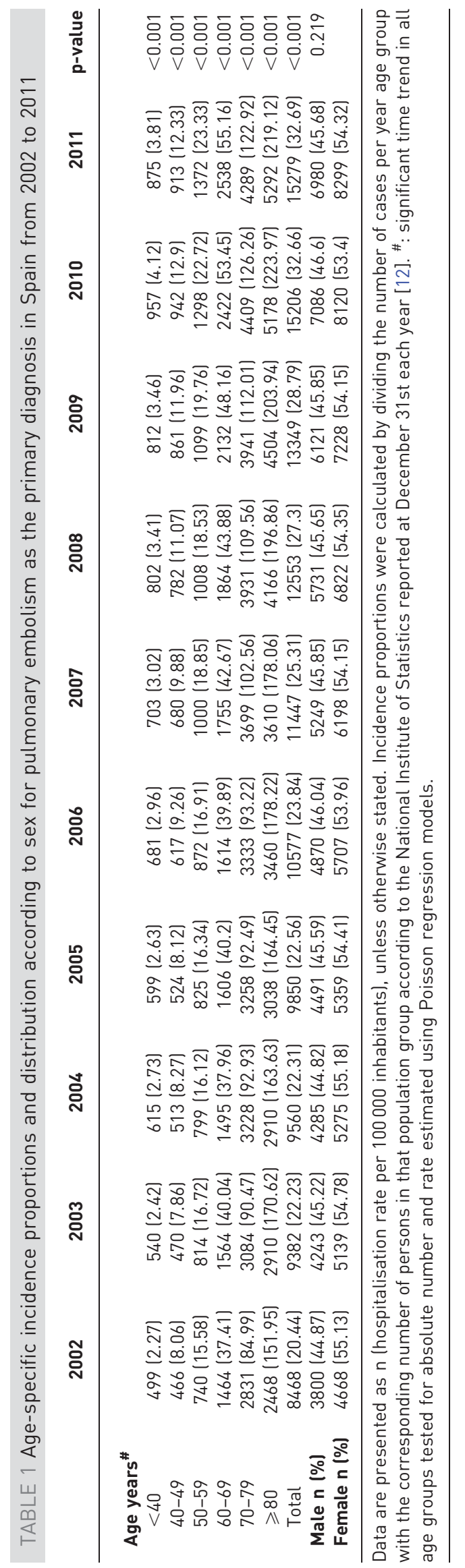

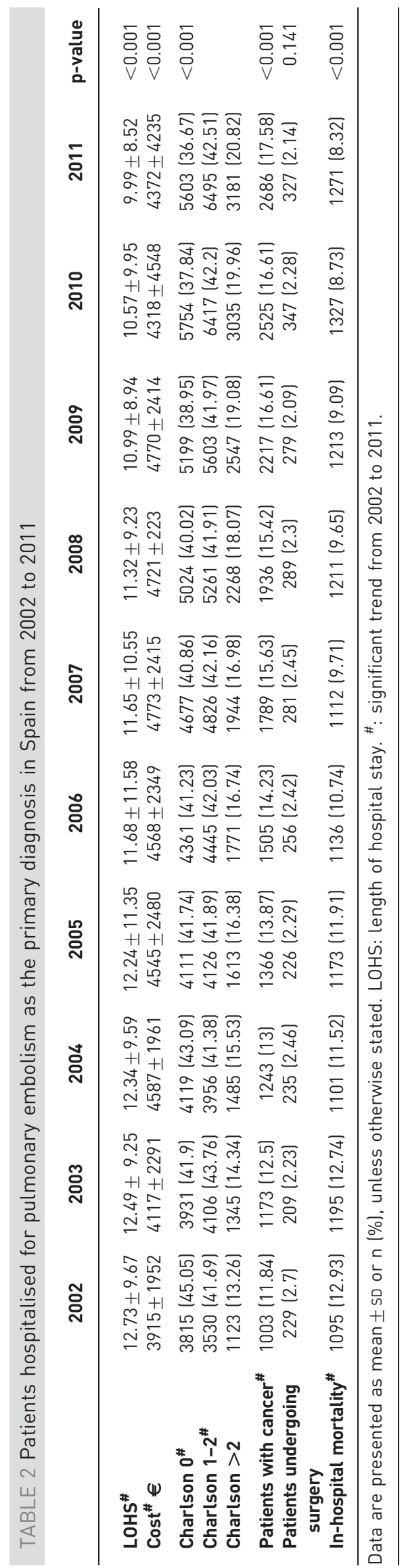




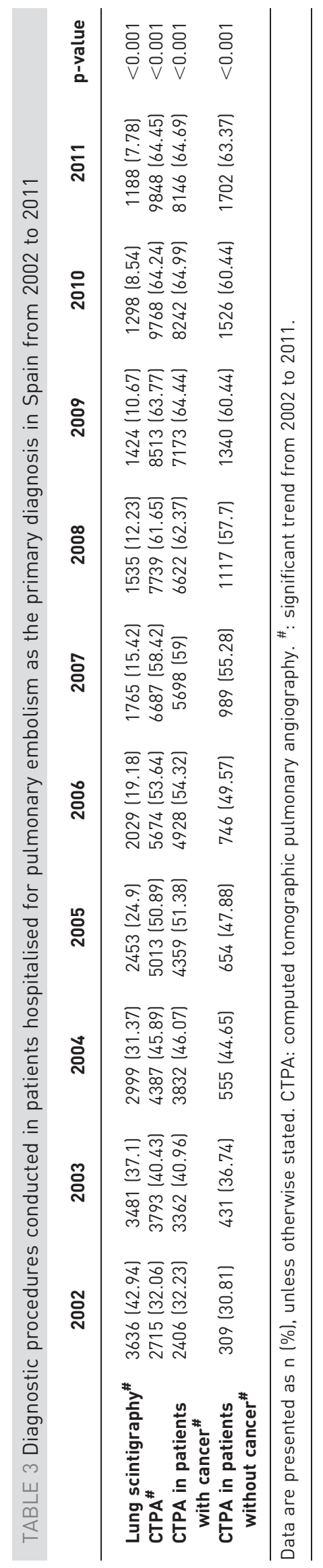

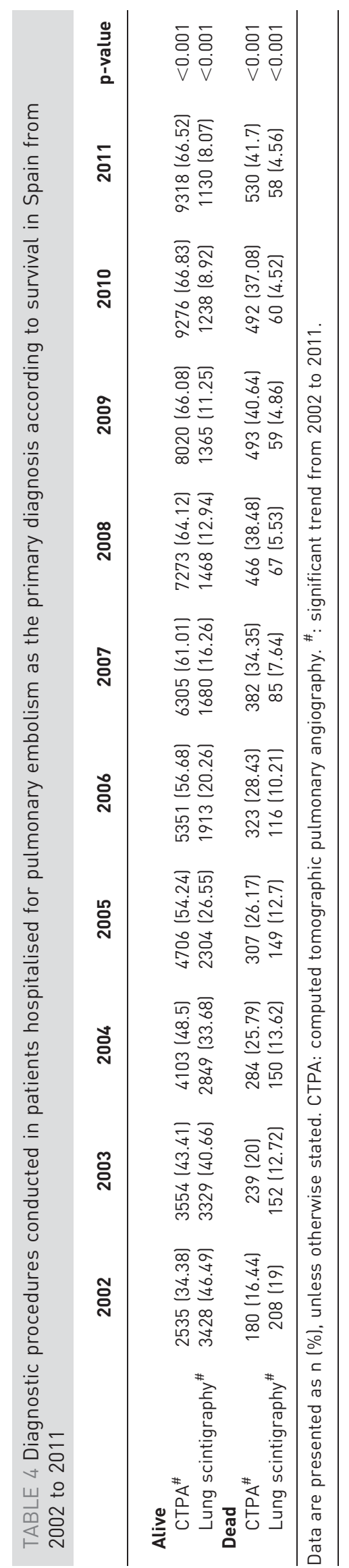


TABLE 5 Multivariate analysis of trends and factors associated with incidence and in-hospital mortality among patients hospitalised for pulmonary embolism as the primary diagnosis in Spain from 2002 to 2011

\section{Incidence \\ IRR $(95 \% \mathrm{CI})^{\#}$}

In-hospital mortality OR $(95 \% \mathrm{CI})^{\circ}$

1
$0.92(0.89-0.96)$
$1.27(1.23-1.31)$
$2.28(2.22-2.34)$
$4.35(4.24-4.46)$
$4.60(4.48-4.71)$
1
$1.18(1.17-1.20)$
1
$1.05(1.04-1.07)$
$1.47(1.46-1.48)$
1
$1.08(1.05-1.12)$
$1.09(1.06-1.12)$
$1.10(1.07-1.14)$
$1.18(1.14-1.21)$
$1.28(1.25-1.32)$
$1.39(1.35-1.43)$
$1.47(1.43-1.51)$
$1.68(1.63-1.72)$
1.69
$1.20(1.64-1.73)$
$0.26(0.25-0.27)$

1

$1.49(1.24-1.80)$

$1.82(1.54-2.16)$

$1.86(1.59-2.19)$

$2.30(1.97-2.68)$

$4.15(3.56-4.83)$

1

$1.06(1.02-1.10)$

1

$1.81(1.72-1.90)$

$4.08(3.86-4.32)$

1

$0.95(0.87-1.05)$

$0.83(0.76-0.92)$

$0.85(0.77-0.94)$

$0.71(0.64-0.78)$

$0.65(0.59-0.72)$

$0.65(0.58-0.71)$

$0.60(0.55-0.66)$

$0.57(0.52-0.62)$

$0.53(0.49-0.59)$

$0.40(0.38-0.41)$

$0.31(0.29-0.34)$

The independent variables included in the models are shown in the table. CTPA: computed tomographic pulmonary angiography. \#: calculated using multivariate Poisson regression for the dependent variable incidence of hospitalisations; ": calculated using logistic regression for the dependent variable in-hospital mortality.

embolism mortality rates over time, although older studies conducted in Spain have demonstrated the opposite trend [9]. In other countries, authors have detected similar changes in the patterns of pulmonary embolism mortality over time. For instance, in the USA and Canada, the mortality rate increased from the $1960 \mathrm{~s}$ through to the early $1980 \mathrm{~s}$, and then decreased until the late $1990 \mathrm{~s}$ [17-20]. After initially increasing [21], mortality rates in the UK steadily declined from the late $1970 \mathrm{~s}$ [22] and in the period between 1996 and 2006 [5]. Otherwise, in Germany, the in-hospital mortality rate ranged from 20.4\% in 2001 to $24.9 \%$ in 2010 , and decreased to $14 \%$ in 2006 [23].

The exact reasons for the discrepancy between the increase in hospital admission rates and the decrease in pulmonary embolism mortality over time are unknown and may warrant further investigation. However, possible explanations have been proposed, including a greater awareness of the incidence of pulmonary embolism in hospitalised patients, an improvement in diagnostic techniques for the identification of this disease [6], more effective treatment, and fewer complications [24]. In this regard, we found a significant increase in the use of CTPA over time, which is associated with an increased incidence of pulmonary embolism and a lower mortality. Indeed, part of this is because unstable patients simply didn't undergo imaging procedures. Another explanation is that CTPA allows pulmonary embolism to be diagnosed earlier and at a less severe stage; therefore, improving the results of the treatment and, as a result, survival. WIENER and co-workers $[4,25]$ have also recently shown a marked increase in the incidence of pulmonary embolism with minimal changes in mortality in the USA with the use of CTPA for diagnosis of pulmonary embolism. The increased use of CTPA may result in over diagnosis and subsequent over treatment, with the detection of potentially harmless pulmonary emboli, which increases pulmonary embolism morbidity but not mortality $[4,25]$. Also, SEGARD et al. [26] found an increase in pulmonary embolism-related imaging in 
recent years, which was driven entirely by increased referrals for CTPA, resulting in more diagnoses but no reduction in deaths from pulmonary embolism in Australia. Other causes have been suggested to explain the divergence between hospital admission rates and pulmonary embolism mortality, such as preventive health interventions, improved clinical awareness of pulmonary embolism risk, improved diagnosis and treatment of venous thromboembolism, and increased use of thromboprophylaxis [6]. However, a decrease in the prevalence of venous thromboembolism risk factors should have led to a parallel reduction in pulmonary embolism hospital admissions rates [27, 28]. Another possible explanation for the decrease in mortality rate is the decrease in autopsy rates in many countries, making death statistics hard to interpret [6].

We observed substantial differences in the incidence and mortality from pulmonary embolism according to age and sex. Increasing age was associated with higher rates of incidence and mortality, a phenomenon that has been reported in previous studies $[5,7,15]$. Also, females had a markedly higher burden of pulmonary embolism incidence and mortality than males, even though males have an increased prevalence of some risk factors, such as cigarette smoking and cancer [27]. FABBIAN et al. [29] have also shown that in-hospital mortality for pulmonary embolism was independently associated with female sex. SHIRAEv et al. [6] detected an increase in pulmonary embolism mortality among young females in Australia, and correlated these results with the effects of pregnancy, smoking and oral contraceptive use. Additionally, the use of hormone replacement therapy may be an important cause of pulmonary embolism in older females [6]. Other authors have also observed an increasing likelihood of in-hospital mortality for first-listed pulmonary embolism in hospitalisations of females compared with males [24]. In any case, the exact reasons for these findings remain unknown and additional research is needed.

Finally, we observed an increased incidence of pulmonary embolism with a higher number of comorbidities. While such comorbidities may predispose patients to develop pulmonary embolism, they may themselves also determine the long-term prognosis of the patients [30]. In fact, NG et al. [31] have recently shown that the Charlson comorbidity index predicts both in-hospital and long-term mortality in patients hospitalised for acute pulmonary embolism. In addition, patients with a Charlson comorbidity index of 0 appear to have a very favourable outcome, with long-term survival similar to the age- and sex-matched general population. Although the outcome data were obtained from a death registry, the results have been extrapolated successfully to other patient groups [32].

Consistent with international trends in length and cost of hospitalisation during this period [15], we observed a shorter length of hospital stay and an increased burden associated with hospital admissions for pulmonary embolism. Although increased comorbidity is one of the factors that may have influenced the cost trend, the reduction in length of hospital stay indicates that the management of the patient with acute pulmonary embolism may have improved in Spain during the study period. Another possibility that may explain the cost trend is that more procedures are performed or the cost of procedures has increased over time. In any case, several studies have shown that the costs per hospitalisation associated with pulmonary embolism events are substantial [23, 33-35].

We should recognise the strengths and limitations of the current study. The main strength lies in a large sample size and standardised methodology, which was constantly maintained during the study period. Nevertheless, our study contains some limitations.

First, a potential source of bias comes from use of ICD-9-CM diagnosis codes to identify patients hospitalised for pulmonary embolism. The major concern of using disease codes in such analyses is of course the questionable accuracy of the primary pulmonary embolism diagnosis. For instance, it is unknown which primary diagnosis code is provided in case of competing diagnosis, i.e. in hospital pulmonary embolism after major surgery. However, this bias does not affect our results meaningfully, as the number of cases of pulmonary embolism secondary to surgery in our study was extremely low ( $\sim 98 \%$ of patients with pulmonary embolism did not recorded any surgical procedure). It is also unknown whether asymptomatic pulmonary embolism accounts for the majority of the excess pulmonary embolism. Demonstration of unsuspected pulmonary embolism occurs in up to 5\% of thoracic computed tomography scans performed for non-pulmonary embolism indications, the majority in the context of malignancy [36]. However, our study has shown that the number of patients receiving thoracic computed tomography scans has increased over time in patients with and without cancer. Therefore, it is expected that the proportion of asymptomatic pulmonary embolism is very low. Furthermore, according to the CMBD methodology, nonsymptomatic incidental findings should not be coded as a diagnosis [13]. Another possible limitation is the certainty of the discharge diagnosis if the patient died during the hospital stay. In this study we have demonstrated that in patients who died in the hospital, imaging tests were also carried out, although in a smaller proportion than in those who survived. In both cases, the use of CTPA increased over time and the use of lung scintigraphy decreased. So, the certainty of the discharge diagnosis is surely higher among survivals. In any case, previous studies have proven that the reliability of the CMBD in the diagnosis of 
venous thromboembolism is high $[37,38]$. Although this is not a valuable validation, we support its use in observational and epidemiological studies. In addition, discharge databases have been used by other authors to assess outcomes, burden and trends of hospital admissions for pulmonary embolism [4, 6, 7, 15, 24].

Secondly, outcomes were limited to the variables coded, which do not include relevant data, such as the severity of the pulmonary embolism or treatment, among others. However, a recent observational study conducted in the USA revealed a decrease in case fatality rate attributable to pulmonary embolism from 1998-2008, which is largely due to a reduction of the case fatality rate in stable patients. There was no reduction of case fatality rate with thrombolytic therapy, although unstable patients who received a combination of vena cava filter with thrombolytic therapy had a relatively low case fatality rate [39]. Therefore, outcomes such as length of hospital stay may have been influenced by other covariates that were different to those we studied. Despite these limitations, the CMBD discharge has the advantage of being mandated by the National Public Health System and it includes almost 100\% of admissions in Spain [40]. In addition, Spain is a large country with a public health system providing full, free of charge medical services to the entire population, so patients come from a variety of socioeconomic categories, improving the external validity of the current results.

In conclusion, the current study provides robust data indicating that, despite increasing hospital admissions for pulmonary embolism over time, in-hospital mortality and length of hospitalisation had decreased but with increasing costs for healthcare resources. These results suggest that the management of acute pulmonary embolism has improved in Spain during the study period.

\section{References}

Sanchez O, Planquette B, Meyer G. Update on acute pulmonary embolism. Eur Respir Rev 2009; 18: 137-147.

2 Edelsberg J, Ollendorf D, Oster G. Venous thromboembolism following major orthopedic surgery: review of epidemiology and economics. Am J Health Syst Pharm 2001; 58: Suppl. 2, S4-S13.

Kearon C. Natural history of venous thromboembolism. Circulation 2003; 107: Suppl. 1, I22-I30.

4 Wiener RS, Schwartz LM, Woloshin S. Time trends in pulmonary embolism in the United States: evidence of overdiagnosis. Arch Intern Med 2011; 171: 831-837.

5 Aylin P, Bottle A, Kirkwood G, et al. Trends in hospital admissions for pulmonary embolism in England: 1996/7 to 2005/6. Clin Med 2008; 8: 388-392.

6 Shiraev TP, Omari A, Rushworth RL. Trends in pulmonary embolism morbidity and mortality in Australia. Thromb Res 2013; 132: 19-25.

$7 \quad$ Yang Y, Liang L, Zhai Z, et al. Pulmonary embolism incidence and fatality trends in Chinese hospitals from 1997 to 2008: a multicenter registration study. PloS One 2011; 11: e26861.

8 Montes Santiago J, Rey García G, Mediero Domínguez A. Tromboembolismo pulmonar en pacientes médicos. Aproximación a los costes hospitalarios y tendencias evolutivas en España [Pulmonary embolism in medical inpatients. An approach to trends and costs in Spain]. An Med Interna 2004; 21: 326-330.

9 Guijarro Merino R, Montes Santiago J, San Román Terán CM. Epidemiología hospitalaria de la enfermedad tromboembólica venosa en España [Epidemiology of venous thromboembolie disease in Spain]. Med Clin (Barc) 2008; 131: Suppl. 2, 2-9.

10 Monreal M, Trujillo-Santos J. Lessons from VTE registries: the RIETE experience. Best Pract Res Clin Haematol 2009; 22: 25-33.

11 El Gobierno de España Ministerio de Empleo y Seguridad Social. Explotación estadística del Conjunto Mínimo Básico de Datos Hospitalarios. Nota metodológica. Norma estatal. www.msssi.gob.es/estadEstudios/estadisticas/ docs/NORMAGRD2011/norma_estatal_2011_notas_metod.pdf Date last updated: January 20, 2014. Date last accessed: September 1, 2013.

12 Instituto Nacional de Estadística (INE). Population estimates. www.ine.es Date last updated: January 20, 2014. Date last accessed: July 22, 2013.

13 Instituto Nacional de la Salud. CMBD Insalud. Análisis de los GRDs. Año 2000. www.ingesa.msc.es/estadEstudios/ documPublica/cmbd2000.htm Date last updated: January 20, 2014. Date last accessed: July 22, 2013.

14 Deyo RA, Cherkin DC, Ciol MA. Adapting a clinical comorbidity index for use with ICD-9-CM administrative databases. J Clin Epidemiol 1992; 45: 613-619.

15 Park B, Messina L, Dargon P, et al. Recent trends in clinical outcomes and resource utilization for pulmonary embolism in the United States: findings from the nationwide inpatient sample. Chest 2009; 136: 983-990.

16 Mellemkjaer L, Sørensen HT, Dreyer L, et al. Admission for and mortality from primary venous thromboembolism in women of fertile age in Denmark, 1977-95. BMJ 1999; 319: 820-821.

17 Horlander KT, Mannino DM, Leeper KV. Pulmonary embolism mortality in the United States, 1979-1998: an analysis using multiple-cause mortality data. Arch Intern Med 2003; 163: 1711-1717.

18 Lilienfeld DE. Decreasing mortality from pulmonary embolism in the United States, 1979-1996. Int J Epidemiol 2000; 29: 465-469.

19 Lilienfeld DE, Chan E, Ehland J, et al. Mortality from pulmonary embolism in the United States: 1962 to 1984. Chest 1990; 98: 1067-1072.

20 Soskolne CL, Wong AW, Lilienfeld DE. Trends in pulmonary embolism death rates for Canada and the United States, 1962-87. CMAJ 1990; 142: 321-324.

21 Butler CM, Cotton LT, Roberts VC. Mortality trends from venous thrombosis and embolism in England and Wales. Lancet 1983; 2: 1314.

22 Goldacre MJ, Roberts S, Yeates D, et al. Hospital admission and mortality rates for venous thromboembolism in Oxford region, UK, 1975-98. Lancet 2000; 355: 1968-1969. 
Kröger K, Küpper-Nybelen J, Moerchel C, et al. Prevalence and economic burden of pulmonary embolism in Germany. Vasc Med 2012; 17: 303-309.

24 Tsai J, Grosse SD, Grant AM, et al. Correlates of in-hospital deaths among hospitalizations with pulmonary embolism: findings from the 2001-2008 National Hospital Discharge Survey. PLoS One 2012; 7: e34048.

25 Wiener RS, Schwartz LM, Woloshin S. When a test is too good: how CT pulmonary angiograms find pulmonary emboli that do not need to be found. BMJ 2013; 347: f3368.

26 Segard T, Macdonald WB. Changing trends in venous thromboembolism-related imaging in Western Australian teaching hospitals, 2002-2010. Med J Aust 2013; 198: 100-103.

27 Zöller B. Time trends in pulmonary embolism: a matter of age and gender. Thromb Res 2013; 132 : 6-7.

28 Burge AJ, Freeman KD, Klapper PJ, et al. Increased diagnosis of pulmonary embolism without a corresponding decline in mortality during the CT era. Clin Radiol 2008; 63: 381-386.

29 Fabbian F, Gallerani M, Pala M, et al. In-hospital mortality for pulmonary embolism: relationship with chronic kidney disease and end-stage renal disease. The hospital admission and discharge database of the Emilia Romagna region of Italy. Intern Emerg Med 2012; 8: 735-740.

30 Lee JW, Cha SI, Jung CY, et al. Clinical course of pulmonary embolism in lung cancer patients. Respiration 2009; 78: 42-48.

31 Ng AC, Chow V, Yong AS, et al. Prognostic impact of the Charlson comorbidity index on mortality following acute pulmonary embolism. Respiration 2013; 85: 408-416.

32 Golpe R, Pérez-de-Llano LA, Castro-Añón O. Prognostic value of the Charlson comorbidity index in pulmonary embolism. Respiration 2013; 85: 438.

33 Spyropoulos AC, Lin J. Direct medical costs of venous thromboembolism and subsequent hospital readmission rates: an administrative claims analysis from 30 managed care organizations. J Manag Care Pharm 2007; 13: 475-486.

34 Fanikos J, Rao A, Seger AC, et al. Hospital costs of acute pulmonary embolism. Am J Med 2013; 126: $127-132$.

35 Kröger K, Küpper-Nybelen J, Moerchel C, et al. Prevalence and economic burden of pulmonary embolism in Germany. Vasc Med 2012; 17: 303-309.

36 Condliffe R, Elliot CA, Hughes RJ, et al. Management dilemmas in acute pulmonary embolism. Thorax 2014; 69: 174-180.

37 Barba R, Losa JE, Guijarro C, et al. Fiabilidad del conjunto mínimo básico de datos (CMBD) en el diagnóstico de la enfermedad tromboembólica venosa [Reliability of minimal basic data set in the diagnosis of thromboembolic disease]. Med Clin (Barc) 2006; 127: 255-257

38 Guijarro R, Montes J, Sanromán C, et al. Venous thromboembolism in Spain. Comparison between an administrative database and the RIETE registry. Eur J Intern Med 2008; 19: 443-446.

39 Stein PD, Matta F, Alrifai A, et al. Trends in case fatality rate in pulmonary embolism according to stability and treatment. Thromb Res 2012; 130: 841-846.

40 Instituto Nacional de la Salud. Conjunto Mínimo Básico de Datos. Hospitales del INSALUD 2001. www.ingesa. msc.es/estadEstudios/documPublica/pdf/CMBD-2001.pdf Date last updated: January 20, 2014. Date last accessed: July 19, 2013 